Open Access

\title{
Adherence to drug treatments and adjuvant barrier repair therapies are key factors for clinical improvement in mild to moderate acne: the ACTUO observational prospective multicenter cohort trial in 643 patients
}

Raúl de Lucas', Gerardo Moreno-Arias², Montserrat Perez-López ${ }^{3}$, Ángel Vera-Casaño ${ }^{4}$, Sonia Aladren ${ }^{5}$, Massimo Milani ${ }^{5 *}$ and on behalf of ACTUO Investigators study group

\begin{abstract}
Background: In acne, several studies report a poor adherence to treatments. We evaluate, in a real-life setting conditions, the impact of compliance to physician's instructions, recommendations and adherence to the treatments on clinical outcome in patients with mild to moderate acne in an observational, non-interventional prospective study carried out in 72 Dermatologic Services in Spain (ACTUO Trial).

Methods: Six-hundred-forty-three subjects were enrolled and 566 patients (88 \%) completed the 3 study visits. Study aimed to evaluate the impact of adherence (assessed with ECOB scale) on clinical outcome, as well as how the use of specific adjuvant treatments (facial cleansing, emollient, moisturizing and lenitive specific topical products) influences treatment's adherence and acne severity ( $0-5$ points score). Recommendation of specific adjuvant skin barrier repair products was made in $85.2 \%$.
\end{abstract}

Results: Overall, clinical improvement was observed throughout follow-up visits with an increased proportion of patients who reported reductions of $\geq 50 \%$ on the total number of lesions (2 months: $25.2 \% ; 3$ months: $57.6 \%$ ) and reductions of severity scores $(2.5,2.0$ and 1.3 at 1,2 and 3 months after treatment, respectively). Adherence to treatment was associated with a significant reduction on severity grading, a lower number of lesions and a higher proportion of patients with $\geq 50 \%$ improvement.

Conclusions: Good adherence to medication plus adherence to adjuvants was significantly associated with a higher clinical improvement unlike those that despite adherence with medication had a low adherence to adjuvants. A good adherence to adjuvant treatment was

associated with improved adherence and better treatment outcomes in mild to moderate acne patients.

(ISRCTN Registry: ISRCTN14257026).

Keywords: Acne vulgaris, Emollients, Observational study, Patient adherence, Topical administration

\footnotetext{
* Correspondence: massimo.milani@isdin.com

${ }^{5}$ Isdin S.A. Medical Department, Provençals 33, Barcelona, Spain

Full list of author information is available at the end of the article
}

(c) 2015 de Lucas et al. Open Access This article is distributed under the terms of the Creative Commons Attribution 4.0 International License (http://creativecommons.org/licenses/by/4.0/), which permits unrestricted use, distribution, and reproduction in any medium, provided you give appropriate credit to the original author(s) and the source, provide a link to the Creative Commons license, and indicate if changes were made. The Creative Commons Public Domain Dedication waiver (http://creativecommons.org/publicdomain/zero/1.0/) applies to the data made available in this article, unless otherwise stated. 


\section{Background}

Acne is a common skin chronic disease that very often requires prolonged treatments [1]. With a chronic course [2] and an episodic pattern of presentation [3], Acne vulgaris lesions occur mainly in exposed areas like the face and the presternal region. The effect of the scarring may be notable and the functional, social and emotional impact on the patient's quality of life may be significant $[4,5]$. Adherence to doctor prescriptions has a major impact on treatment outcome [6]. Several studies conducted over the past few years suggest that adherence to acne medications is particularly low $[3,7]$ and has been associated with inadequate response to therapy [8], especially when both topical and systemic treatments are prescribed to the same patient [9]. The treatment plan and the choice of specific active ingredients should take into account not only the individual characteristics of the patient and/or the disease but also their preferences and expectations with treatment as well as questions of convenience [10].The adverse effects of the various therapies available should also be borne in mind in order to increase adherence to the physician's instructions and recommendations, thus promoting what are known as health-related behaviours [7]. Specific systemic and topical acne treatments are very often associated with local side effect such as dry, burning and itching sensations [7] hence poor tolerability further worsens compliance [11].'Based on the evidence and clinical experience obtained with topical treatments and their possible irritant effects [12], adjuvant skin barrier repair therapies such as specific emulsion and detergent products, hydrating and emollients are often prescribed in order to reduce these side effects, all in search of improving adherence to therapeutic strategies [13, 14]. Other factors such as a good and efficient physician-patient relationship are also crucial for improving adherence to therapeutic strategy [15] since this interaction includes specific roles and motivations [16] that might be essential to the healing of many patients, particularly so for patients with chronic disease or disease having a negative impact on quality of life and self-esteem such as acne [17]. Adherence with acne therapies has been evaluated mainly in subjects irrespective to grading severity of the condition (from mild to severe forms) $[8,18]$ but, so far, few data are available regarding this conduct in patients with mild to moderate forms. We evaluated in a real-life setting condition the impact of adherence to dermatologist instructions and recommendations and adherence to the treatments $x$ (specific anti acne treatments and adjuvant therapies) on the clinical outcome in patients with mild to moderate acne. The participating physicians of the ACTUO trial were practicing experienced Spanish dermatologists working in hospital outpatient dermatology services.

\section{Methods}

ACTUO was a multicenter, prospective, observational, epidemiological study of patients with mild to moderate acne treated at 72 dermatology services throughout Spain between October 2011 and November 2012. All study materials were evaluated and approved by a Clinical Research Ethics Committee before the study start. Approval (Ethics Committee of "Centro Medico Teknon" Barcelona Spain) was obtained August 1, 2011.

\section{a) Subjects}

A total of 643 subjects with mild to moderate acne, after they provided written informed consent, were enrolled in the study. Eligibility criteria were men and women with mild to moderate acne vulgaris eligible for a specific acne treatment (topical retinoid agents or antiseptics and/or systemic antibiotics) willing to participate in the study. The study was conducted at three visits carried out in a period of three months (V1: baseline, and two follow-up visits: V2 and V3). In all 3 visits, data on acne severity was recorded on the Case Report Form (CRF) by the dermatologist using the global ranking system of the FDA [19] (0: no lesions, 4: severe) and the total number of acne lesions. Acne severity was also evaluated by the patient. In addition, adherence to anti-acne treatments and adjuvant therapies was also assessed trough study.

\section{b) Primary outcome}

The main objective of this analysis was to evaluate the impact of adherence to the treatments on the clinical outcome in patients with mild to moderate acne. A secondary outcome was to evaluate the impact of the use of specific adjuvant treatments (facial cleansing products, emollient moisturizing and lenitive specific topical cream products containing mainly rhamnosoft as emollient and antiflammatory agent) on adherence level and entity of the clinical outcome obtained. Acne severity was assessed at each visit with a 5-point score system (from 0 to 4 ), absolute count of lesions and percentage of patient reaching a $\geq 50 \%$ reduction in lesion numbers. Adherence to treatment was evaluated at visit 2 and visit 3 by means of validated 4-item questionnaire (ECOB) with a dichotomous classification: good adherence $($ ECOB score $=4$ ) and poor adherence $(E C O B<4)$. Poor adherence to treatment was defined as a different to expected answer on the ECOB questionnaire. The four questions of ECOB were according to Pawin et al. [20].

The degree of adherence to the adjuvant measures prescribed by the doctor (application of cleansers and moisturizers for facial skin care) was assessed using the data provided by the patients attending all three study visits. It was considered that patients who followed all their doctor's 
instructions and recommendations in at least three of the four assessments showed compliance with the medical advice. The effects of adherence to adjuvant treatment on the doctor's and patient's final assessments of acne severity was compared in patients "without / practically without lesions" (FDA scores $=0-1$ ) and patients "with lesions" (FDA scores $\geq 2-4$ ). The ACTUO study has evaluated also the impact of acne and acne treatments on quality of life evaluated by means of Cardiff Acne Disability Index [21] (CADI) and Dermatology Life Quality Index (DLQI) [22]. However these data would be presented elsewhere.

\section{c) Statistical methods}

The FDA ratings made by the physician and patient after three months were grouped into two categories: presence of lesions (FDA $\geq 2-4$ ), or absence or near absence of lesions $($ FDA $=0-1)$. Using the parameters severity and total number of lesions, improvement was assessed by classifying patients into two groups based on the reduction of acne severity ( $\geq 50 \%$ improvement vs. improvement $<50 \%$ ) and the percentages of reduction of comedones and papules/ pustules. Statistical analysis was performed using the statistical software SPSS ver.19. Continuous variables were expressed as mean (SD). Categorical binary variables were expressed as proportions (\%). The Mann-Whitney, Wilcoxon and the chi-square tests were used for inference statistical analysis tests. A multivariate logistics regression analysis was performed in order to assess the correlation between improvement of acne severity score, percentage of patients with $50 \%$ or more in acne lesion number reduction, and the following variable: demographic data, adherence to specific treatments and to adjuvant treatments.

\section{Results}

Table 1 gives information about patients' characteristics at the time of enrollment. A total of 643 cases were enrolled. A total of 566 patients $(88 \%)$ completed the 3-visit study. Data are presented as per protocol analysis. At baseline, adjuvant products were prescribed in $83.8 \%$ of the patients. The application frequency was $1.3 /$ day. Prescription for specific adjuvant products was made for $85.2 \%$ of the patients. Severity of acne assessed by the physician was $2.5 \pm 0.6$ and mean number of comedones was $18.9 \pm 2$. Severity acne score was significantly $(p<0.001)$ reduced in comparison with baseline after 1 month $(2.0 \pm 0.8)$ and after 3 months $(1.3 \pm 0.9)$. At the end of study period patients scoring 0 (complete cure of acne) were $17 \%$. In general, significant reductions in the severity scores vis-àvis the baseline visit were observed in both doctor's and patients' assessment $(2.1 \pm 0.9$ at one month and $1.4 \pm 0.9$ at three months; $p<0.001$ ). Figure 1 shows the evolution of acne severity score in the population as a whole.
Percentage of patients showing at least $50 \%$ of lesion reduction was $25.2 \%$ at visit 2 and $57.6 \%$ at visit 3 .

According to the ECOB scores, good adherence to treatment was documented in $50.0 \%$ of the patients at visit 2 and in $66.3 \%$ at visit 3 . Good adherence to treatment was associated with a significant $(p<0.05)$ acne improvement in comparison with poor adherence group at both control visits. At visit 3 acne severity score was $1.19 \pm 0.8$ in patients with good adherence to adjuvants vs. $1.4 \pm 0.9$ in poor adherence group. Good adherence to specific acne treatments (facial cleansers) was documented in $83.6 \%$ at visit 2 and in $89.9 \%$ at visit 3 . At the end of study, patients with good adherence to treatment presented a significant improvement of acne both in term of acne severity score (1.2 vs. $1.7 ; p=0.001)$ and regarding the proportion of patients whom reduction of lesions was $\geq 50 \%$ (60.9 \% vs. $32.8 \% ; p<0.001)$ when compared to those with poor adherence (Fig. 2). In patients for whom the dermatologist had prescribed adjuvant therapies, a good adherence to this treatment was documented in $36.2 \%$. Adherence to adjuvant treatment improves acne thus it was associated with a significant reduction of score grading severity at visit 3 (1.2 vs. $1.4 ; P=0.002)$, with a higher percentage of reduction of average lesions number vs. baseline (50.8 \% vs. $43.7 \% ; p=0.015)$ and with more patients obtaining a $\geq 50 \%$ reduction in acne lesions' number ( $65.9 \%$ vs. $51.5 \% ; p=0.003$ ) in comparison with the low adherence group (Fig. 2). Adherence to adjuvant treatments was associated with a greater proportion of patients with a complete cure of acne (defined as no or very few acne lesions) at the final visit in comparison with poor-adherers ( $66.5 \%$ vs. $52.6 \% ; p=0.004)$. In particular, good adherence to adjuvant treatments improved the adherence to topical retinoid therapy therefore influencing clinical improvement of acne showing a higher proportion patients with adherence to treatment in adherers to adjuvants when compared to non-adherers $(85.5 \%$ vs. $70.7 \% ; p<0.001)$. In addition, in patients with a good adherence toadjuvants were significantly more frequently considered with an $\geq 50 \%$ improvement on clinical outcome in comparison with those that did not adhere to adjuvants ( $65.9 \%$ vs. $51.5 \% ; p=0.003$ ).

Multiple logistic regression analysis was performed to identify factors influencing clinical improvement of acne. Adherence to specific treatments, adherence to adjuvant treatments and good adherence to treatment are significantly $(p=0.001)$ correlated with clinical improvement of acne. In accordance with previous studies, sex (women vs. men) and the fact to be accompanied (by parents/tutor or relative) or not at the medical visit are also independent factors affecting improvement clinical outcome of acne. Other analysis allowed observing an association between the patient's assessment of quality of life based on the DLQI scale and the adherence to adjuvant treatment since rates of improvement 
Table 1 Sociodemographic data and acne severity at baseline visit

\begin{tabular}{|c|c|c|c|c|}
\hline & & $n$ & mean (SD) & n (\%) \\
\hline \multirow[t]{2}{*}{ Gender } & Male & 635 & - & $240(37.8 \%)$ \\
\hline & Female & & & $395(62.2 \%)$ \\
\hline Age & & 617 & $21.3(7.2)$ & \\
\hline \multirow[t]{8}{*}{ Study level } & Primary school & 632 & - & $24(3.8 \%)$ \\
\hline & Secondary school & & & $151(23.9 \%)$ \\
\hline & Vocational training (school) & & & $51(8.1 \%)$ \\
\hline & Pre-university & & & $188(29.7 \%)$ \\
\hline & Vocational training (technical college) & & & $30(4.7 \%)$ \\
\hline & First cycle university studies & & & $41(6.5 \%)$ \\
\hline & Second cycle university studies & & & $146(23.1 \%)$ \\
\hline & Others & & & $1(0.2 \%)$ \\
\hline \multirow{5}{*}{$\begin{array}{l}\text { Physician's acne } \\
\text { evaluation }\end{array}$} & Without lesions & 640 & $2.47(0.64)$ & - \\
\hline & Practically without lesions & & & $32(5.0 \%)$ \\
\hline & Mild & & & $298(46.6 \%)$ \\
\hline & Moderate & & & $290(45.3 \%)$ \\
\hline & Severe & & & $20(3.1 \%)$ \\
\hline \multirow{5}{*}{$\begin{array}{l}\text { Patient's acne } \\
\text { evaluation }\end{array}$} & Without lesions & 594 & $2.10(0.85)$ & $16(2.7 \%)$ \\
\hline & Practically without lesions & & & $126(21.2 \%)$ \\
\hline & Mild & & & $245(41.2 \%)$ \\
\hline & Moderate & & & $195(32.8 \%)$ \\
\hline & Severe & & & $12(2.0 \%)$ \\
\hline \multirow[t]{5}{*}{ Lesion location ${ }^{a}$} & Facial & 637 & - & $622(97.6 \%)$ \\
\hline & Back & & & $212(33.3 \%)$ \\
\hline & Shoulders & & & $81(12.7 \%)$ \\
\hline & Presternal area & & & $73(11.5 \%)$ \\
\hline & Others & & & $2(0.3 \%)$ \\
\hline \multirow{3}{*}{$\begin{array}{l}\text { Total number of lesions }{ }^{\text {a }} \\
\text { (Percentages of } 568 \\
\text { patients with lesions) }\end{array}$} & Comedones & 546 & $18.9(20.4)$ & 532 (93.7 \%) \\
\hline & Papules/pustules & 551 & $11.4(10.2)$ & $545(96.0 \%)$ \\
\hline & Nodules & 378 & $0.9(1.7)$ & 147 (25.9\%) \\
\hline \multirow{2}{*}{$\begin{array}{l}\text { Recommendation of } \\
\text { specific adjuvant } \\
\text { products (facial } \\
\text { soaps/cleansers and } \\
\text { specific facial care products) }\end{array}$} & No & 640 & - & $104(16.3 \%)$ \\
\hline & Yes & & & $536(83.8 \%)$ \\
\hline
\end{tabular}

on QoL were lower in non-compliers (43\% vs. $56.1 \%$, $p=0.007$ ).

\section{Discussion}

Low adherence to treatments is still a relevant problem in the treatment of mild to moderate acne [23]. The definition of strategies to improve adherence with both pharmacological and behavioural treatment remains a major challenge. Suboptimal medication adherence is one of the major reasons for treatment failure in subject with acne vulgaris [24]. Our findings reveal that dermatologists in
Spain frequently prescribe specific adjuvant treatment for acne. They recommend the use of specific products including non-comedogenic soaps and moisturizers in over $80 \%$ of acne patients. The synergy between the effects of drug treatment and adjuvant products can improve comfort during the various stages of treatment and can encourage patients to continue the application of the products. Adherence to adjuvant treatment was associated not only with a 2.4-fold increase in the probability of adherence to pharmacological treatment, but also with a significant reduction in acne severity, in the number of lesions as assessed by both 


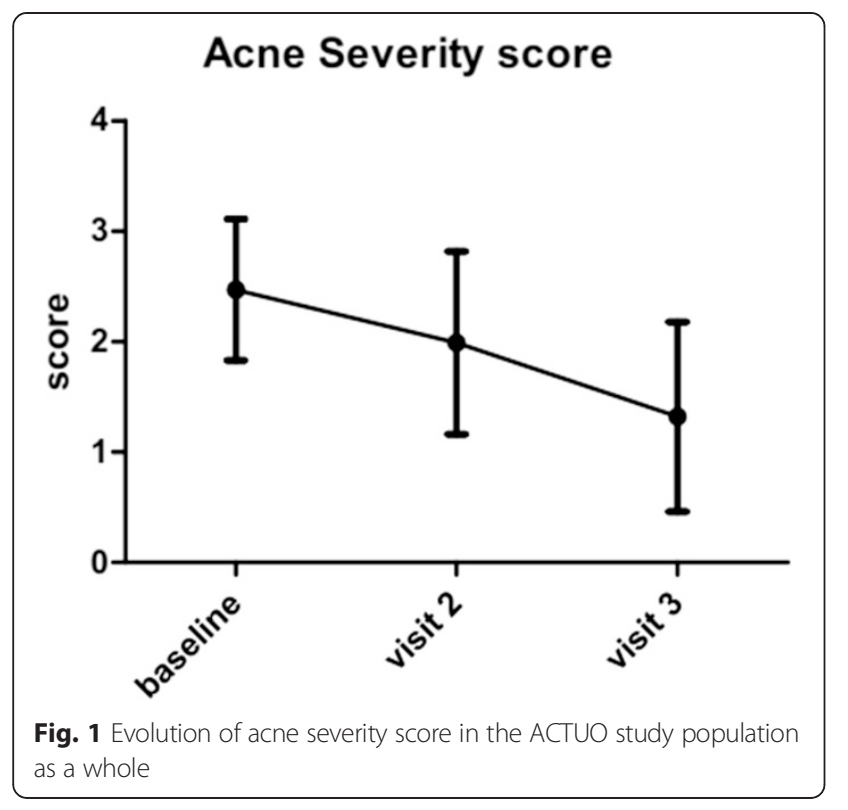

the treating physician and the patient, and a higher percentage of patients whose severity improved by $50 \%$ or more. In the ACTUO study low adherence to treatment was found in $50 \%$ of the patients (at 1 month) and this result is in line with previous published data. Low adherence to adjuvant treatments was observed in up to $63.8 \%$ of subjects after 3 months. In addition we have to consider that these data derived from a per protocol analysis, not including $12.4 \%$ of patients not attending to control visits which therefore could be considered as"non-complier" by definition. In line with previous experiences [3], we have observed that adherence to pharmacological treatment is a key factor for the improvement of acne both in terms of reduction of severity grading, number of lesions and percentage of patients obtaining at least a $50 \%$ acne improvement. Good adherence to adjuvant treatments also improves clinical evolution of acne with a greater reduction of severity of grading and number of lesions and with a significant greater percentage of patients obtaining a $50 \%$ or more improvement of acne in comparison with patients with poor adherence to adjuvant treatments. Furthermore, patients with good adherence with drug and those with adherence to adjuvant treatments presented the highest percentage of patients with improvement of acne lesions $\geq 50 \%$. In addition, in the present study it was shown that adherence to adjuvant products is the second most important factor (just after adherence to drug treatment) influencing the degree of acne improvement. A good adherence to adjuvant treatment is associated with an improved adherence to acne treatments and with a better treatment outcome in mild to moderate acne patients. Some limitations should be taken in account in evaluating the results of our study. In particular the evaluation of compliance was based on self-reporting questionnaire. This approach however was used in other studies assessing the same outcome and the ECOB questionnaire is considered a validated tool $[3,18]$. On the other hand, we believe that the sample size and the study design adopted, quite close to "real life" conditions of acne treatment strategies, could be considered as aspects increasing the external validity and the generalization of the observed results of the ACTUO.

\section{Conclusions}

The ACTUO observational study results confirm that adherence to pharmacological treatment and adjuvant therapies are both key factors of acne improvement in terms of reduction of severity, number of lesions and percentage of patients with at least a $50 \%$ acne improvement. In addition,

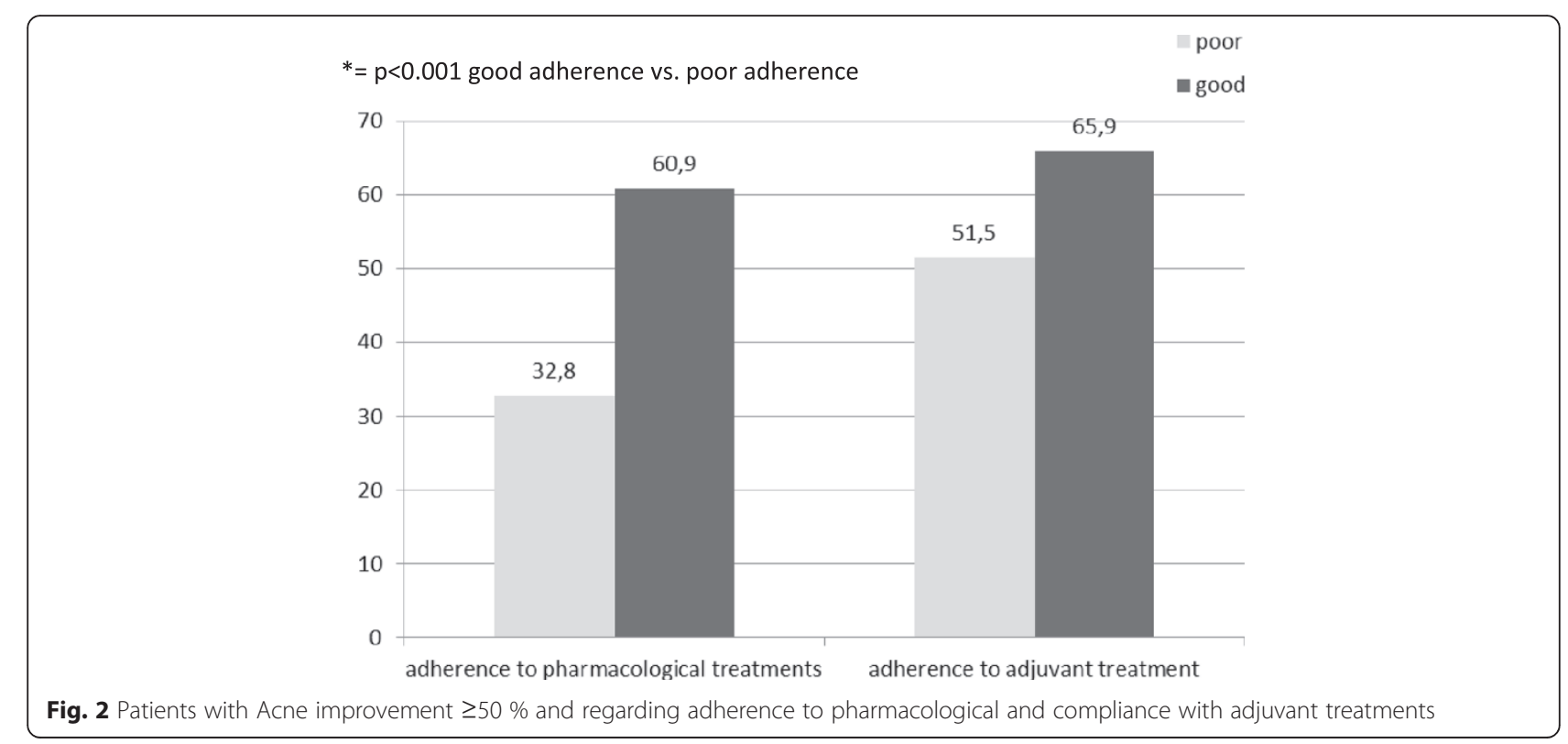


adherence to adjuvant treatment with specific cleansers and moisturizers for patients with acne is the second most important factor in achieving symptom improvement, after completion of drug treatment. Good adherence to adjuvant treatment is associated with a 2.2-fold increase in the probability of adherence to topical pharmacological treatment and with significant reductions in the severity and number of acne lesions.

\section{Abbreviations}

ECOB: Elaboration de un outil de evaluación de l'observance des traitaments medicamenteux; CRF: Case report form; FDA: Federal Drugs Administration.

\section{Competing interests}

Sonia Aladren and Massimo Milani are ISDIN employees. The ACTUO study was supported by an unrestricted grant of ISDIN SA.

\section{Authors' contributions}

RDL, GMA, MPL, AVC participated in the study design, coordination and patients enrollment. MM and SA participated in the design of study protocol and data analysis. All authors read and approved the final manuscript.

\section{Acknowledgements}

Actuo Study Group Investigators: Abellaneda Fernández, Cristina; Aguayo Leyva, Ingrid Rocío; Alcaraz León, Inmaculada; Alonso García, Ignacio; Arechalde Pérez, Ana; Balbín Carrero, Eva; Ballestero Corominas, Alejandro; Bilbao Badiola, Ibon; Boada García, Aram; Ciudad Blanco, Cristina; De Lucas, Raúl; Del Pozo Hernando, Luis Javier; Del Rio Reyes, Rosa; Eguino Gorrochategui, Patricia; Escalas Taberner, Juan; Espelt Otero, Jorge Luis; Fernández Angel,Isabel; Fernández Casado, Alex; Fernández Torres, Rosa Ma; Gallego Álvarez, Silvia; Galvany Rosell, Loida; Gamo Villegas, Reyes; Ginarte Val, Manuel; Gómez Fernández, Cristina; Gómez Vázquez, Mercedes; Gutiérrez De La Peña, Javier; Hernandez Cano, Natalia; Houmani Houmani, Manmoud; Ibargoyen Esnal, Jesús; Iglesias Sancho, Maribel; Izquierdo Herce, Noelia; Jeremías Torruella, Javier; Latorre Fuentes, José Ma; Llambrich Mañes, Alex; López Ferrer, Ana; Mariscal Polo, Amaia; Márquez Balbas, Gemma; Martin Ezquerra, Gemma; Martin Urda, Maria Teresa; Martinez Escala, Maria Estela; Martinez Fernández, Matilde; Mieras Barceló, Caterina; Molina Ruiz, Ana; Montero Pérez, Iria; Moreno Arias, Gerardo A.; Nadal Llado, Cristina; Naranjo Díaz, Maria José; Nasarre Calvo,Jaume; Navajas Pinedo, Belén; Oleaga Morante, José Manuel; Perelló Llinas, Guillermo; Pérez Losada, Ma Eugenia; Pérez-López, Montserrat; Pérez-Beato De Cos, Ma Paz; Pestoni Porven, Carmela; Poza Magdalena, Olga; Rocamora Duran, Vicenc; Rodriguez Caruncho, Clara; Rodriguez Granados, Ma Teresa; Roe Crespo, Esther; Ruiz Carrascosa, José Carlos; Salgado Boquete, Laura; Sanchez Muros, Virginia; Sanz De Galdeano Palacio,Carmen; Taberner Ferrer, Rosa; Trasobares Marugan, Lidia; Uria García, Ma Carmen Valle Martin, Ma Del Mar; Valle Santana, Pilar; Vázquez García, Juan; Velasco Pastor, Manuel; Vera Casaño, Angel; Vilarrasa Rull, Eva; Waadad Waadad, Tamim.

\section{Author details}

${ }^{1}$ Hospital Universitario La Paz, Madrid, Spain. ${ }^{2}$ Hospital Quirón Teknon, Barcelona, Spain. ${ }^{3}$ Clínica Dermatológica de Moragas, Barcelona, Spain. ${ }^{4}$ Hospital Carlos Haya, Málaga, Spain. ${ }^{5}$ Isdin S.A. Medical Department, Provençals 33, Barcelona, Spain.

Received: 16 April 2015 Accepted: 7 September 2015 Published online: 11 September 2015

\section{References}

1. Kligman AM. An overview of acne. J Invest Derm. 1974;62:268-87.

2. Bhate K, Williams HC. Epidemiology of acne vulgaris. Br J Dermatol. 2013;168:474-85.

3. Thiboutot D, Gollnick H, Bettoli V, Dréno B, Kang S, Leyden JJ, et al. New insights into the management of acne: an update from the Global Alliance to Improve Outcomes in Acne group. J Am Acad Dermatol. 2009;60:51-50.

4. Lasek RJ, Chren MM. Acne vulgaris and the quality of life of adult dermatology patients. Arch Dermatol. 1998;134:454-8.
5. Goulden V. Guidelines for the management of acne vulgaris in adolescents. Pediatr Drugs. 2003;5:301-13.

6. Weiden PJ, Rao N. Teaching medication compliance to psychiatric residents: placing an orphan topic into a training curriculum. Acad Psychiatry. 2005;29:203-10.

7. Haider A, Shaw JC. Treatment of acne vulgaris. JAMA. 2004:292:726-35.

8. McDonald HP, Garg AX, Haynes RB. Interventions to enhance patient adherence to medication prescriptions: scientific review. JAMA. 2002;288:2868-79.

9. Dréno B, Layton A, Zouboulis CC, López-Estebaranz JL, Zalewska-Janowska A, Bagatin E, et al. Adult female acne: a new paradigm. J Eur Acad Dermatol Venereol. 2013;27:1063-70.

10. Russell JJ. Topical therapy for acne. Am Fam Physician. 2000;61:357-65.

11. Snyder S. Medical adherence to acne therapy: a systematic review. Am J Clin Dermatol. 2014;15:87.

12. Weiss JS. Current options for the topical treatment of acne vulgaris. Pediatr Dermatol. 1997;14:480-8.

13. Herane MI, Fuenzalida H, Zegpi E, De Pablo C, Espadas MJ, Trullás C, et al. Specific gel-cream as adjuvant to oral insotretinoin improved hydration and prevented TEWL increase - a double blind, randomized, placebo-controlled study. J Cosmet Dermatol. 2009:8:181-5.

14. Del Rosso JQ. Clinical relevance of skin barrier changes associated with the use of oral isotretinoin: the importance of barrier repair therapy in patient management. J Drugs Dermatol. 2013;12:626-31.

15. Renzi C, Picardi A, Abeni D, et al. Association of dissatisfaction with care and psychiatric morbidity with poor treatment compliance. Arch Dermatol. 2002;138:337-42.

16. Tsou AY, Creutzfeldt CJ, Gordon JM. The good doctor: professionalism in the 21st century. Handb Clin Neurol. 2013;118:119-32.

17. Farin E, Gramm L, Schmidt E. Predictors of communication preferences in patients with chronic low back pain. Patient Prefer Adherence. 2013;7:1117-27.

18. Zaghloul SS, Cunliffe WJ, Goodfield MJ. Objective assessment of compliance with treatments in acne. Br J Dermatol. 2005;152:1015-21.

19. US Department of Health and Human Services Food and Drug Administration Center for Drug Evaluation and Research (CDER). Guidance for Industry; AcneVulgaris: Developing Drugs for Treatment. 2005.

20. Pawin H, Beylot C, Chivot M, Faure M, Poli F, Revuz J, et al. Creation of a tool to assess adherence to treatments for acne. Dermatology. 2009;218:26-32.

21. Motley RJ, Finlay AY. Practical use of a disability index in the routine management of acne. Clin Exp Dermatol. 1992;17:1-3.

22. De Tiedra AG, Mercadal J, Badía X, Mascaró JM, Herdman M, Lozano R. Adaptación transcultural al español del cuestionario Dermatology Life Quality Index (DLQI): El índice de calidad de vida en dermatología. Actas Dermatosifilogr. 1998;89:692-700.

23. Jones-Caballero M, Pedrosa E, Peñas PF. Self-reported adherence to treatment and quality of life in mild to moderate acne. Dermatology. 2008;217:309-14

24. Miyachi Y, Hayashi N, Furukawa F, Akamatsu H, Matsunaga K, Watanabe S, et al. Acne management in Japan: study of patient adherence. Dermatology. 2011;223:174-81.

\section{Submit your next manuscript to BioMed Central and take full advantage of:}

- Convenient online submission

- Thorough peer review

- No space constraints or color figure charges

- Immediate publication on acceptance

- Inclusion in PubMed, CAS, Scopus and Google Scholar

- Research which is freely available for redistribution 